\title{
Fenotipo de hipercolesterolemia familiar definitivo con estudio genético negativo en Argentina
}

\author{
Phenotype of definite familial hypercholesterolemia with negative genetic study \\ in Argentina
}

\author{
Pablo Corral ${ }^{1 *}$, Virginia Bañares², Benjamín Sáenz ${ }^{3}$, Valeria Zago ${ }^{4}$, Agustina Sarobe $^{1}$, Graciela López ${ }^{4}$, \\ Gabriela Berg 4 y Laura Schreier ${ }^{4}$
}

${ }^{1}$ Facultad de Medicina, Cátedra de Farmacología, Universidad FASTA, Mar del Plata; ${ }^{2}$ Departamento de Genética Experimental, Centro Nacional de Genética Médica "Dr. Eduardo Castilla", Administración Nacional de Laboratorios e Institutos de Salud "Dr. Carlos Malbrán", Buenos Aires; ${ }^{3}$ Departamento de Investigación, Universidad FASTA, Mar del Plata; "Departamento de Bioquímica Clínica, Facultad de Farmacia y Bioquímica, Laboratorio de Lípidos y Aterosclerosis, Universidad de Buenos Aires, Buenos Aires, Argentina

\begin{abstract}
Resumen
Objetivo: La hipercolesterolemia familiar (HF) es una enfermedad monogénica asociada a variantes en los genes RLDL, APOB y PCSK9. El diagnóstico inicial se basa en criterios clínicos, como el de la red de clínica de lípidos holandesa (DLCN). Un puntaje > 8 puntos califica al paciente como "definitivo" para diagnóstico de HF. La identificación de una variante en estos genes permite realizar el cribado en cascada familiar y caracterizar mejor al paciente en cuanto al pronóstico y el tratamiento. Métodos: En el marco del Programa de Detección de HF en Argentina (Estudio Da Vinci) se evaluó a 246 pacientes hipercolesterolémicos, 21 con puntaje DLCN > 8 (diagnóstico definitivo). Se estudió a estos pacientes con secuenciación de próxima generación para reconocer variantes genéticas, con un panel ampliado de 23 genes, sumado al análisis de grandes rearreglos y por último se aplicó un score poligénico de 10 SNP (polimorfismo de nucleótido único) relacionados con aumento del c-LDL. Resultados: De los 21 pacientes, 10 presentaron variantes en RLDL, uno en APOB junto a APOE, uno en LIPC más puntaje poligénico elevado, dos pacientes con una deleción y una duplicación en RLDL y este último caso con una variante en LIPA. Es destacable que 6 de los 21 pacientes con puntaje DLCN > 8 no mostraron ninguna alteración genética. Conclusiones: El 28\% de los pacientes con diagnóstico clínico definitivo de HF no evidenció alteración genética. Las posibles explicaciones de este resultado serían la presencia de mutaciones en nuevos genes, los efectos confundidores del ambiente sobre los genes o la interacción gen-gen y por último la imposibilidad de detectar variantes con la metodología actual disponible.
\end{abstract}

Palabras clave: Hipercolesterolemia Familiar. Diagnóstico genético. Score poligénico.

\begin{abstract}
Objective: Familial hypercholesterolemia $(F H)$ is a monogenic disease, associated with variants in the LDLR, APOB and PCSK9 genes. The initial diagnosis is based on clinical criteria like the DLCN criteria. A score $>8$ points qualifies the patient as "definite" for FH diagnosis. The detection of the presence of a variant in these genes allows carrying out familial cascade
\end{abstract}

Correspondencia:

*Pablo Corral

E-mail: drpablocorral@gmail.com
Fecha de recepción: 14-05-2019

Fecha de aceptación: 19-11-2019

DOI: 10.24875/ACM.19000221
Disponible en internet: 29-01-2020 Arch Cardiol Mex. 2020;90(2):151-157 www.archivoscardiologia.com 1405-9940/@ 2019 Instituto Nacional de Cardiología Ignacio Chávez. Publicado por Permanyer. Este es un artículo open access bajo la licencia CC BY-NC-ND (http://creativecommons.org/licenses/by-nc-nd/4.0/). 
screening and better characterizes the patient in terms of prognosis and treatment. Methods: In the context of the FH detection program in Argentina (Da Vinci Study) 246 hypercholesterolemic patients were evaluated, 21 with DLCN score $>8$ (definite diagnosis). These patients were studied with next generation sequencing to detect genetic variants, with an extended panel of 23 genes; also they were adding the large rearrangements analysis and a polygenic score of 10 SNP (single nucleotide polymorphism) related to the increase in LDL-C. Results: Of the 21 patients, 10 had variants in LDLR, 1 in APOB with APOE, 1 in LIPC plus elevated polygenic score, and 2 patients showed one deletion and one duplication in LDLR, the later with a variation in LIPA. It is highlighted that 6 of the 21 patients with a score $>8$ did not show any genetic alteration. Conclusions: We can conclude that $28 \%$ of the patients with definite clinical diagnosis of FH did not show genetic alteration. The possible explanations for this result would be the presence of mutations in new genes, confusing effects of the environment over the genes, the gene-gene interactions, and finally the impossibility of detecting variants with the current available methods.

Key words: Familial Hypercholesterolemia. Genetic diagnosis. Polygenic score.

\section{Introducción}

La hipercolesterolemia familiar (HF) es una enfermedad monogénica, autosómica dominante, asociada con variantes en los genes del receptor de la lipoproteína de baja densidad $(R L D L)$, de la apolipoproteína $\mathrm{B}$ $(A P O B)$ y de la proproteína convertasa subtilisina/kexina tipo 9 (PCSK9) 1 .

Los pacientes portadores de esta patología se presentan a menudo en forma asintomática con valores de c-LDL > $200 \mathrm{mg} / \mathrm{dl}$ o con episodios cardiovasculares precoces ( $<50$ años en varones y $<60$ años en mujeres). Las características de su herencia permiten identificar a familiares afectados y a múltiples integrantes en una misma familia con niveles elevados de C-LDL o eventos cardiovasculares ${ }^{2}$.

El diagnóstico inicial se basa en diferentes criterios clínicos que evalúan los antecedentes cardiovasculares personales, familiares y estigmas clínicos característicos, como los xantomas tendinosos y el arco corneal temprano, y por último los valores plasmáticos de c-LDL. Los criterios de la red de clínicas de lípidos holandesas (DLCN, dutch lipid clinic criteria network) son los más utilizados. Un valor $>8$ puntos califica al paciente como "definitivo" para el diagnóstico de $\mathrm{HF}^{3}$.

Si se considera que la herencia es autosómica dominante y que la forma heterocigota de la HF es efecto de una variante monogénica única en uno de los tres genes primarios relacionados con $\mathrm{HF}$ en las siguientes proporciones: $95 \%$ de $R L D L, 3 \%$ de $A P O B$ y $1 \%$ de PCSK9, además de la mayor disponibilidad en el acceso a las técnicas modernas de secuenciación, el diagnóstico genético es una herramienta central en el diagnóstico de la $\mathrm{HF}^{4}$.

Luego de verificar la ausencia de una variación o mutación puntual en algunos de estos tres genes es imperativo descartar la presencia de los denominados grandes rearreglos, fundamentalmente, duplicaciones o deleciones, con técnicas de biología molecular o bioinformática, los cuales no pueden detectarse en la secuenciación génica. Distintos reportes muestran que $60 \%$ a $80 \%$ de los pacientes con diagnóstico definitivo de HF por criterios clínicos, presentan variantes genéticas relacionadas con la $\mathrm{HF}^{4-7}$.

La verificación de la presencia de una variante en los genes vinculados con HF permite a su vez realizar la detección en cascada en el resto de los familiares y caracterizar mejor al paciente en cuanto al pronóstico y tratamiento ${ }^{8}$.

En los pacientes con fenotipo compatible con HF y sin variantes detectadas, las causas poligénicas explican la mayor parte de los casos. Diferentes grupos de investigadores han desarrollado cálculos para obtener un puntaje a partir de polimorfismos de nucleótidos únicos (SNP) relacionados con el aumento de los valores circulantes de C-LDL ${ }^{5,9}$. Por último, es posible señalar otras causas potenciales de casos fenotípicamente compatibles con HF sin hallazgos genéticos, entre ellas las raras mutaciones en otros genes menores relacionados con el metabolismo lipídico, como APOE, ABCG5, ABCG8, LIPA, CYP27A1 o STAP1 y las mutaciones o variantes inaccesibles con secuenciación exónica ${ }^{10}$.

El objetivo del presente trabajo consiste en la evaluación de un grupo de pacientes con fenotipo y diagnóstico definitivo de HF y resultado genético negativo, luego de un análisis biomolecular completo y ampliado.

\section{Material y métodos}

Se evaluó a 246 pacientes hipercolesterolémicos incluidos durante el año 2016 en el Programa De Detección De Hipercolesterolemia Familiar en la Argentina 
(Estudio Da Vinci), llevado a cabo en el Partido de General Pueyrredón, Provincia de Buenos Aires. Los participantes registraron niveles de colesterol > $300 \mathrm{mg} / \mathrm{dl}$ o c-LDL > $190 \mathrm{mg} / \mathrm{dl}$ y otorgaron su consentimiento informado para su evaluación clínica, bioquímica y genética. El estudio recibió aprobación del Comité de Ética, Res CD FFyB Res CD 1762/17.

De cada paciente se obtuvo su historial familiar y personal, con especial foco en enfermedad cardiovascular aterosclerótica (angina de pecho, infarto de miocardio, cirugía de revascularización miocárdica, accidente cerebrovascular y/o enfermedad vascular periférica o carotídea), hospitalizaciones, hipertensión, diabetes mellitus y tratamiento hipolipemiante actual. Se efectuó una cuidadosa exploración física, con especial dedicación en la detección de xantomas tendinosos o arco corneal. Cuando estaban disponibles, se obtuvieron estos datos clínicos de familiares de primer grado (padres, hijos y hermanos). Se consideró enfermedad cardiovascular prematura como la presencia de alguna de las manifestaciones de enfermedad cardiovascular aterosclerótica ya mencionadas previamente, antes de los 55 años para hombres y 65 para mujeres. Se aplicaron los criterios DLCN, considerando el mayor valor de c-LDL registrado $^{3}$. Un total de $40 \%$ de pacientes recibían tratamiento hipolipemiante y, cuando no se disponía del valor máximo de c-LDL sin tratamiento, se aplicó una corrección basada en dosis y tipo de estatina ${ }^{11}$.

No se incluyeron a 21 sujetos por los siguientes criterios de exclusión aplicados: $n=8$ presentaron un valor de TSH > $10 \mathrm{mUI} / \mathrm{L}, 4$ con creatinina $>1.7 \mathrm{mg} / \mathrm{dl}$, 2 con fosfatasa alcalina $>200 \mathrm{U} / \mathrm{L}$, un paciente con triglicéridos $>1,000 \mathrm{mg} / \mathrm{dl}$, 3 mujeres excluidas por embarazo y 3 pacientes que se opusieron a la toma de muestra de sangre. Por lo tanto, finalmente se incluyeron al final a 225 sujetos con potencial HF.

Se extrajeron las muestras de sangre en estado sin ayuno, las cuales se remitieron al Laboratorio de Lípidos y Aterosclerosis de la Universidad de Buenos Aires para el estudio bioquímico. Se determinaron en suero colesterol total, triglicéridos, c-HDL, c-LDL directo, creatinina y fosfatasa alcalina mediante métodos enzimáticos estandarizados (Roche Diagnostics, Mannheim, Alemania) con un autoanalizador Cobas C-501. El promedio de los coeficientes de variación (CV) intraanálisis para estos parámetros fue $<2.3 \%$ y CV interanálisis $<3.0 \%$. La apoproteína $B$ se determinó por método inmunoturbidimétrico en el mismo autoanalizador, con un CV interanálisis $<2.5 \%$. Se midió TSH por quimioluminiscencia (DPC, Immulite, Los Ángeles, CA, EE.UU.) con CV intraanálisis e interanálisis $<3.5 \%$.
En todos los sujetos con puntaje de DLCN $>8$ se efectuó el estudio genético. De la muestra de sangre entera se extrajo DNA de los pacientes en el Centro Nacional de Genética Médica ${ }^{12}$. El estudio genético se llevó a cabo en el Boston Heart Diagnosis, Massachusetts, EE.UU., mediante secuenciación de nueva generación descrito en el estudio previo ${ }^{13}$ con el fin de identificar variantes genéticas en los genes clásicos relacionados con c-LDL elevado: RLDL, APOB, PCSK9, y en otros genes asociados con dislipidemias: $L D L-$ RAP1, STAP1, ABCG5, ABCG8, APOE, LIPA, CYP27A1, LIPC, LIPG, y DHCR24. Además, se estudió un panel de 10 variantes comunes (SNP: polimorfismo de nucleótido único) en los siguientes genes: rs6544713 en ABCG8, rs515135 en APOB, rs12740374 en CELSR2, rs3846663 en HMGCR, rs2650000 en HNF1, rs6511720 en $R L D L$, rs6102059 en MAFB, rs10401969 en NCAN, rs11206510 en PCSK9, yrs1501908 en TIMD4. A partir de la detección de los SNPs se calculó el puntaje de riesgo genético (PRG) con el fin de evaluar la contribución poligénica del C-LDL elevado ${ }^{14}$. Por último, se efectuó análisis bioinformático de variaciones en el número de copias (VNC) para identificar los grandes rearreglos en la estructura de genes que no se detectan por la secuenciación genética ${ }^{15}$.

Los datos se expresan como mediana y rango y porcentaje. El análisis estadístico se llevó a cabo con SPSS 19.0 .

\section{Resultados}

Veintiún de los 225 pacientes estudiados con hipercolesterolemia registraron un puntaje de DLCN $>8$, en un intervalo de 9 a 14. De estos 21 pacientes con diagnóstico clínico definitivo de HF, dos tuvieron familiares de primer grado con enfermedad cardiovascular, una mediana (rango) de c-LDL de $250 \mathrm{mg} / \mathrm{dl}$ (181-431), que correspondía al valor máximo registrado eventualmente 0 corregido al final por el tratamiento recibido. Nueve pacientes tuvieron arco corneal antes de los 45 años, ocho xantomas tendinosos, tres enfermedad coronaria prematura y dos enfermedad arterial cerebral prematura. El resto del perfil lipídico demostró una mediana (rango) de triglicéridos: $121 \mathrm{mg} / \mathrm{dl}$ (62-314); c-HDL: 50 mg/dl (36-68); y ApoB: 164 mg/dl (91-278).

Diez de los 21 pacientes con fenotipo definitivo de HF mostraron variantes puntuales en $R L D L$ y uno en $A P O B$, junto con una variante en $A P O E$, lo que constituyó un $52.4 \%$ de pacientes que poseen variantes puntuales en genes clásicos. El estudio del panel ampliado de genes en los pacientes con fenotipo definitivo reveló también 
un caso con mutación en $L I P C$, uno con variante en LIPA y otro en CYP27A1. En la tabla 1 se muestran las variantes detectadas, así como su clasificación con respecto a su patogenicidad.

El estudio bioinformático de VNC a posteriori para detectar grandes rearreglos en la estructura del gen demostró que uno de los casos con estudio genético negativo y el portador de la variante genética en el gen LIPA eran portadores de una deleción de los exones 16-18 y una duplicación de los exones 2-8, respectivamente, en el gen RLDL (Tabla 1).

Con respecto al cálculo del puntaje de riesgo genético, 5 de los 21 pacientes presentaron un PRG mayor al valor de corte de 1.96 establecido en el percentil $90^{\circ}$, aunque tales casos también eran portadores de variantes en RLDL $(\mathrm{n}=2)$ y en los casos con variantes en APOB/APOE, CYP27A1 y LIPC. Cabe destacar que $6(28 \%)$ de los 21 pacientes con puntaje de DLCN $>8$ no mostraron ningún tipo de alteración genética luego del estudio genético ampliado y de la detección de causas poligénicas (Fig. 1).

\section{Discusión}

La HF es una enfermedad monogénica, con alta penetrancia, asociada clásicamente a variantes en los genes RLDL, APOB y PCSK91. La amplia disponibilidad actual de los estudios genéticos, en parte por la reducción de los costos, ha permitido profundizar el estudio de los pacientes con fenotipo de HF y realizar una correlación entre su sospecha clínica y los resultados de la secuenciación de los genes potencialmente participantes en el metabolismo del colesterol.

Luego de la sospecha clínica de HF, el procedimiento que sigue es la aplicación de un puntaje clínico o criterios diagnósticos para establecer el diagnóstico clínico de la HF. Los criterios clínico-bioquímicos más utilizados son los DLCN, donde en base a cinco ítems se concluye si el paciente tiene un diagnóstico definitivo (> 8 puntos), probable (6-8), posible (3-5) o improbable $(<3)^{3}$.

El análisis genético, en busca de la variante genética, es el paso posterior, siempre que se tengan los recursos e instrumentos necesarios para realizar la secuenciación ${ }^{15}$.

La identificación de la variante genética permite caracterizar mejor al paciente; las diferentes mutaciones orientan acerca del pronóstico de la enfermedad; y es posible realizar un tamizaje o cascada familiar y, en casos seleccionados, apoyar una terapéutica específica ${ }^{16,17}$.

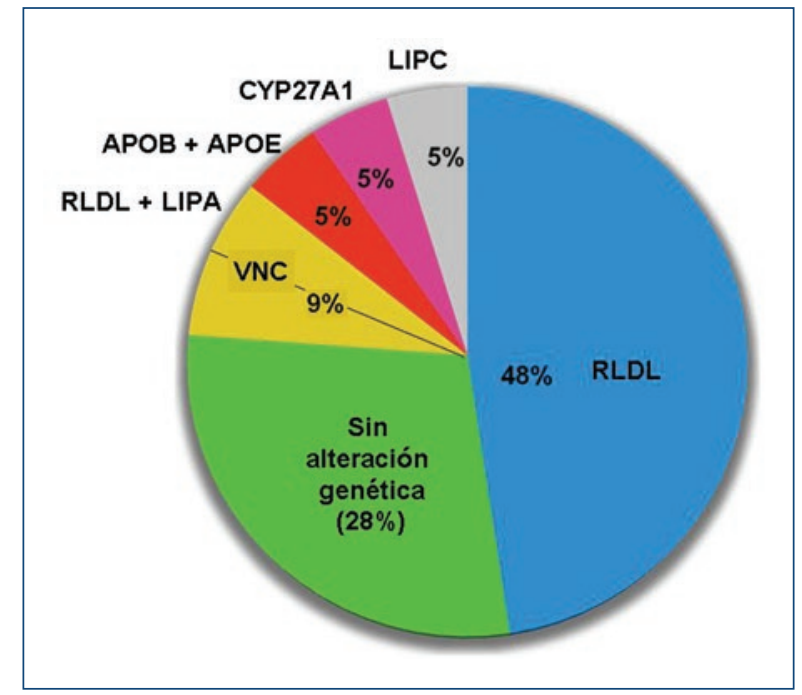

Figura 1. Distribución de las variantes genéticas detectadas en los 21 pacientes con puntaje DLCN $>8$. CNV: variaciones en el número de copias; $R L D L$, gen del receptor de las LDL; LIPC: gen de la lipasa C; CYP27A1: gen del citocromo P450 familia 27 tipo A1; $A P O B$ : gen de la apolipoproteína b; APOE: gen de la apoproteína E; LIPA: gen de la lipasa $A$.

Durante el desarrollo del Estudio Da Vinci (Estudio Argentino Prevalencia en Hipercolesterolemia Familiar) se evaluó a 246 pacientes con niveles elevados de colesterol y/o c-LDL, de los cuales 21 registraron un puntaje de DLCN > 8, es decir, un diagnóstico definitivo de HF. Se estudiaron a estos pacientes con secuenciación genética para la detección de variantes monogénicas, con un panel ampliado de 23 genes, sumado al análisis de grandes rearreglos; por último se aplicó puntaje poligénico (PRG) de 10 SNP relacionados con aumento de C-LDL. De los 21 pacientes, 10 presentaron variantes en $R L D L$, uno en $A P O B$ junto a $A P O E$, uno en LIPC y uno en CYP27A1, éstos también con puntaje poligénico elevado, y dos pacientes con una deleción y una duplicación (rearreglos genéticos) en $R L D L$, este último con una variante en LIPA.

Cabe destacar que seis de los 21 pacientes con puntaje $>8$ no mostraron ninguna alteración genética. Por lo tanto, este $28 \%$ de los pacientes comprende la categoría de sujetos con fenotipo de HF y estudio genético negativo, teniendo en cuenta que en el presente estudio el panel genético evaluado se amplió e incluyó causas poligénicas, lo cual valoriza aún más el hallazgo ${ }^{18}$.

Las posibles explicaciones a este hallazgo serían las siguientes: a) existencia de variaciones o mutaciones en genes aún no reconocidos como causante de HF; b) presencia de mutaciones en regiones no detectadas por las 





tecnologías disponibles en la actualidad como las mutaciones mapeadas en zonas intrónicas profundas o promotoras distales; c) efectos del medioambiente sobre los genes (fenómenos epigenéticos), d) interacciones entre diferentes genes en un mismo individuo ${ }^{19-21}$.

Por otro lado, la validación en esta población del puntaje DLCN para diagnóstico clínico, así como PRG, es necesaria para confirmar su utilidad en el medio y asegurar la especificidad y sensibilidad diagnóstica ${ }^{10,14}$.

La implementación del panel ampliado de genes más allá de los típicos relacionados con HF ha permitido reconocer variantes inusuales relacionadas con hipercolesterolemias severas ${ }^{13}$. En este estudio, un paciente mostró una variante en el gen de la enzima ácida lisosomal (LIPA), cuya deficiencia se ha vinculado con dislipemia y mortalidad prematura ${ }^{22}$. Este paciente es un doble heterocigoto, ya que también mostró una duplicación en $R L D L$; en cualquier caso, la variante en LIPA es de significancia incierta. Otras variantes en genes infrecuentes que se identificaron en los pacientes con diagnóstico definitivo de HF se detectaron en el gen de la lipasa hepática (LIPC) y en CYP27A1 relacionado con la xantomatosis cerebrotendinosa. Sin embargo, esas variantes también coexistieron con PRG elevado, lo que señala causas poligénicas. Por lo tanto, queda por dilucidar la alteración que ocasiona mayormente la elevación de c-LDL, ya que pueden coexistir causas poligénicas $o$ variantes en genes inusuales cuya patogenicidad aún es incierta ${ }^{10}$.

La utilidad actual de los estudios genéticos en cuanto a la toma de decisión terapéutica no está establecida. Resultaría de utilidad en casos de pacientes con HF homocigota, con objeto de cuantificar la actividad residual del receptor LDL, pero no en la mayoría de los casos de HF. Por lo tanto, el nivel de c-LDL y el riesgo global del paciente deben ser los factores determinantes en cuanto a la conducta terapéutica en los pacientes con $\mathrm{HF}^{18,20}$.

Puede concluirse que, a pesar de una búsqueda exhaustiva y ampliada de las causas genéticas en pacientes con diagnóstico clínico definitivo de HF, el 28\% (6/21) de los pacientes no evidenció alteración genética. Futuros y más complejos estudios, como la realización de exomas a diferentes integrantes de una misma familia, quizá terminen de demostrar y explicar los hallazgos referidos.

\section{Conclusión}

Puede afirmarse que dentro de la cohorte analizada en una región específica de la Provincia de Buenos Aires, el $28 \%$ de los pacientes con diagnóstico clínico de HF (DLCN) no tuvo alteración genética alguna, luego de realizar un análisis extenso, no sólo de las variantes en genes comunes relacionadas con HF, sino también del estudio de genes considerados no clásicos y de la aplicación de un PRG a fin de evaluar la contribución poligénica del c-LDL elevado y, por último, del análisis bioinformático para identificar grandes rearreglos en la estructura de genes no reconocidos por secuenciación genética.

\section{Agradecimientos}

Los autores expresan su agradecimiento a los doctores Ernst Schaefer, Eliana Polisecki, Andrew Geller (Boston Heart Diagnostics, Framingham, MA, EE.UU.) y Robert Hegele (Robarts Research Institute, London, Ontario, Canadá) por la ejecución del estudio genético y los asesoramientos recibidos.

\section{Financiación y conflicto de intereses}

Este trabajo se llevó a cabo con subsidio irrestricto de Amgen Biotechnology Company, CA, EE.UU. y subsidio de la Universidad de Buenos Aires, UBACYT 20020170100259BA; Pablo Corral: Amgen Biotechnology Company y Sanofi Pharmaceutical Company; Laura Schreier: Sanofi Pharmaceutical Company.

\section{Responsabilidades éticas}

Protección de personas y animales. Los autores declaran que los procedimientos seguidos se conformaron a las normas éticas del comité de experimentación humana responsable y de acuerdo con la Asociación Médica Mundial y la Declaración de Helsinki.

Confidencialidad de los datos. Los autores declaran que han seguido los protocolos de su centro de trabajo sobre la publicación de datos de pacientes.

Derecho a la privacidad y consentimiento informado. Los autores han obtenido el consentimiento informado de los pacientes o sujetos referidos en el artículo. Este documento obra en poder del autor de correspondencia.

\section{Bibliografía}

1. Nordestgaard BG, Chapman MJ, Humphries SE, Ginsberg HN, Massana L, Descamps OS, et al. Familial hypercholesterolaemia is underdiagnosed and undertreated in the general population: guidance for clinicians to prevent coronary heart disease: Consensus Statement of the European Atherosclerosis Society. Eur Heart J 2013:34:3478-3490.

2. Gidding SS, Champagne MA de Ferranti SD, Defesche J, Ito MK, Knowles JW, et al. The agenda for familial hypercholesterolemia. Circulation 2015;132:2167-2192. 
3. World Health Organization. Familial hypercholesterolaemia: report of a second WHO Consultation, World Health Organization, Geneva, Switzerland,1999. WHO publication No. WHO/HGN/FH/CONS/99.2.

4. Hegele RA, Ban MR, Cao H, Mclntyre AD, Robinson JF, Wang J. Target next- generation secuencing in monogenis dyslipidemias. Curr Op Lipido 2015:26:103-13

5. Wang J, Dron JS, Ban MR, Robinson JF, McIntyre AD, Alazzam M, et al. Polygenic versus monogenic causes of hypercholesterolemia ascertained clinically. Arterioscler Thromb Vasc Biol 2016;36:2439-2445.

6. Sarraju A, Knowles JW. Genetic testing and risk scores: impact on familial hypercholesterolemia. Front Cardiovasc Med 2019; 29;6:5

7. Sharifi M, Futema M, Nair D, Humphries SE. Genetic architecture of familial hypercholesterolaemia. Curr Cardiol Rep 2017;19 (5):44.

8. Lázaro P, Pérez de Isla L, Watts GF, Alonso R, Muñiz O, Fuentes F, et al. Cost-effectiveness of a cascade screening program for the early detection of familial hypercholesterolemia. J Clin Lipidol 2017;11(1):260-271.

9. Talmud PJ, Shah S, Whittall R, Futema M, Howard P, Cooper JA, et al. Use of low-density lipoprotein cholesterol gene score to distinguish patients with polygenic and monogenic familial hypercholesterolaemia: a case-control study. Lancet 2013;381:1293-1301.

10. Hooper AJ, Burnett JR, Bell DA, Watts GF. The present and the future of genetic testing in familial hypercholesterolemia: opportunities and caveats. Curr Atheroscler Rep 2018;20(6):31.

11. Haralambos K, Whatley SD, Edwards R, Gingell R, Townsend D, Ashfield-Watt $\mathrm{P}$, et al. Clinical experience of scoring criteria for familial hypercholesterolaemia $(\mathrm{FH})$ genetic testing in Wales. Atherosclerosis 2015;240:190-196.

12. Miller SA, Dykes DD, Polesky HF. A simple salting out procedure for extracting DNA from human nucleated cells. Nucleic Acids Res 1988;16 (3):1215.
13. Corral P, Geller AS, Polisecki EY, López GI, Bañares VG, Cacciagiu L, et al. Unusual genetic variants associated with hypercholesterolemia in Argentina. Atherosclerosis 2018;277:256-261.

14. lacocca MA, Hegele RA. Recent advances in genetic testing for familial hypercholesterolemia. Expert Rev Mol Diagn 2017;17 (7):641-651.

15. lacocca MA, Wang J, Dron JS, Robinson JF, Mclntyre AD, Cao H, et al. Use of next-generation sequencing to detect LDLR gene copy number variation infamilial hypercholesterolemia. JLipid Res2017:58(11):2202-2209.

16. Berberich AJ, Hegel RA, The complex molecular genetics of familial hypercholesterolaemia. Nat Rev Cardiol 2018. https://doi.org/10.1038/ s41569-018-0052-6.

17. Sturn AC, Knowlwes JW, Gidding SS, Amad ZS, Ahmed CD, Ballantayne CM, et al. Genetic testing for familial hypercholesterolemia: JACC Scientific Expert Panel. J Am Cardiol 2018;72(6):662-680.

18. Santos RD. Phenotype vs genotipe in severe familial hypercholesterolemia: What matters for the clinician? Curr Op Lipidol 2017;28(2):130-135.

19. Guay SP, Brisson D, Lamarche B, Gaudel D, Bouchard L. Epipolymorphisms within lipoprotein genes contribute independently to plasma lipid levels in familial hypercholesterolemia. Epigenetics 2014;9(5):718-29.

20. Santos RD, Guidding SS, Hegele RA, Cuchel MA, Barter PJ, Watts GE, et al. Defining severe familial hypercholesterolaemia and the implications for clinical management a consensus statement from the Intenational Atherosclerosis Society Severe Familial Hypercholesterolemia Panel. Lancet Diabetes Endocrinol 2016;4(10):850-61.

21. Futema M, Plagnol V, Li K, Whittall RA, Neil HA. Whole exome sequencing of familial hypecholesterolaemia patients negative for LDLR/APOB/ PCSK9 mutations. J Med Genet 2014;51:537-544.

22. Chora JR, Alves AC, Medeiros AM, Mariano C, Loberinhas G, Guerra A et al. Lysosomal acid deficiency: a hidden disease among cohorts of familial hypercholesterolemia? J Clin Lipidol 2017;11(2):477-484.e2. 